\title{
Transferencia y erosión de patrones melódicos en bilingües de español e inglés en California
}

\author{
Sergio Robles-Puente* \\ West Virginia University, EE.UU.
}

\begin{abstract}
Resumen
Las situaciones de contacto entre el español y el inglés han motivado en numerosas ocasiones el estudio de procesos de transferencia y variación en múltiples niveles de la gramática. Sin embargo, estos fenómenos no han recibido atención suficiente al nivel de la entonación. Este estudio analiza las producciones de veinticuatro bilingües divididos en cuatro grupos con diferentes grados de exposición al español y al inglés con el objetivo de buscar asimetrías y similitudes entonativas tanto entre hablantes como entre lenguas. El análisis de 488 movimientos tonales en enunciados declarativos, imperativos y vocativos muestra que el inglés y el español comparten diversas melodías. Asimismo, los hablantes bilingües pueden presentar variaciones por transferencia o erosión en sus inventarios tonales independientemente de su afinidad e identificación con uno de los idiomas. Dichas variaciones son fruto de diferentes tipos de exposición a cada lengua.
\end{abstract}

Palabras clave: entonación, bilingüismo, español, inglés, transferencia.

* Para correspondencia, dirigirse a: Sergio Robles-Puente (seroblespuente@mail.wvu. edu), Department of World Languages, Literatures \& Linguistics - West Virginia University. Chitwood Hall, PO Box 6298. Morgantown, WV 26506 (USA). 


\title{
TRANSFER AND EROSION OF MELODIC PATTERNS \\ in Spanish-English bilinguals in California
}

\begin{abstract}
Contact situations between Spanish and English have motivated the study of transfer and variation processes at different levels of the grammar in multiple occasions. Nevertheless, these phenomena have not been studied in detail at the intonational level. This research analyzes the productions of twenty-four bilinguals divided into four groups with different levels of exposure to English and Spanish with the objective of looking for asymmetries and similarities between speakers and languages. The analysis of 488 tonal movements in statements, imperatives and vocatives show that Spanish and English may share multiple melodies. At the same time, bilingual speakers may present modifications due to transfer or attrition processes in their tonal inventories regardless of their affinity and identification with one of the languages. These modifications are the result of different kinds of exposure to each language.
\end{abstract}

Keywords: intonation, bilingualism, Spanish, English, transfer.

Recibido: 17/02/18 Aceptado: $13 / 01 / 19$

\section{INTRODUCCIÓN ${ }^{1}$}

El contacto de lenguas provee un marco idóneo para el estudio de modificaciones a cualquier nivel de la estructura gramatical (fonología, morfología y sintaxis - Thomason y Kaufman 1988) y el caso del español en contacto con el inglés no es una excepción. Silva-Corvalán (1994), en uno de los primeros estudios de esta índole, destaca que, aunque la estructura de la lengua es la que controla la introducción de nuevos elementos en el

\footnotetext{
1 Este trabajo ha sido realizado gracias a la ayuda institucional concedida por el Ministerio de Ciencia, Innovación y Universidades del Gobierno de España, Agencia Estatal de Investigación, Plan Nacional de I+D+i, al Proyecto de Investigación GEOPROSODICEstudio geoprosódico y sociodialetal del español americano septentrional, Ref. FFI201568508-P (2015-2019), dirigido por la IP Yolanda Congosto Martín (http://www.geoprosodic. com). Mi más sincero agradecimiento a todos los participantes que lo han hecho posible, así como a los colegas que han revisado las versiones previas de este artículo.
} 
sistema lingüístico, es la historia de los hablantes la que puede determinar la dirección y la relevancia de esos cambios. La autora demuestra que este es el caso de la sintaxis y la morfología del español en la comunidad mexicana de Los Ángeles y menciona como ejemplos la posición de los clíticos, el orden Sujeto-Verbo y la falta de uso del complementante 'que'. De una forma similar, Lipski (2008) argumenta que los hablantes bilingües de español e inglés en las comunidades mexicanoamericanas presentan características gramaticales que se alejan de lo que consideramos un español estándar. Estas incluyen el uso de inflexiones nominales y adjetivales incorrectas, errores en las conjugaciones verbales y en el uso de preposiciones, fallos con los artículos definidos e indefinidos o un uso redundante de pronombres de sujeto. Aunque las observaciones de estos estudios sirven como ejemplo de cambios morfológicos y sintácticos, en los últimos años también ha habido un creciente interés por las modificaciones en el nivel fonético-fonológico y más particularmente a nivel prosódico.

En lo que respecta al ritmo prosódico, son numerosos los estudios que resaltan cómo los hablantes bilingües de español e inglés en comunidades de EE.UU. pueden presentar características más o menos similares a las de los monolingües, dependiendo de la generación a la que pertenecen y de sus historiales lingüísticos (ver Fought 2003, Carter 2005, Shousterman 2014, Carter y Wolford 2016). En el caso de la entonación, diversos estudios han identificado modificaciones melódicas en el español en contacto con el inglés, así como con otras lenguas. Por ejemplo, Elordieta (2003), Colantoni y Gurlekian (2004) y O’Rourke (2005) muestran cómo la posición por defecto del pico tonal en acentos tonales prenucleares de frases declarativas en español puede verse alterada por contacto con el euskera, el italiano y el quechua, respectivamente. Robles-Puente (2012) encuentra que el euskera y el español de Gernika (País Vasco, España) comparten tonos de frontera bajos en frases absolutas, de la misma forma que Alvord (2006) identifica tonos de frontera bajos en el mismo tipo de frases tanto en el español de Cuba como en el de Miami. Finalmente, Thomas y Ericson (2007) y Miglio et al. (2013) muestran cómo los mexicanoamericanos y los hablantes de español chicano tienen propiedades entonativas diferentes a los anglohablantes y a los hablantes de español mexicano respectivamente.

El presente estudio pretende aportar más información sobre cómo el dominio prosódico de la frase entonativa puede verse afectado en situaciones en las que coexisten dos gramáticas (Selkirk 1980, 1984, Nespor y Vogel 1986, Hayes 1989, Schiering et al. 2007, Prieto 2006). Asimismo, los resultados del estudio pueden capturar modificaciones melódicas del español en contacto con el inglés para así complementar la lista existente de descripciones entonativas de variedades del español (e inglés). Para ello nos 
concentramos en analizar la entonación de hablantes bilingües de español e inglés en la comunidad mexicana de Los Ángeles (California, EE.UU.).

\section{PARTICIPANTES}

Veinticuatro participantes bilingües fueron seleccionados para participar en el estudio. Con la excepción del grupo control de inglés, todos los participantes eran hablantes nativos de español mexicano. La razón para escoger participantes con este perfil en Los Ángeles atendía a varias razones. En primer lugar, el $47.7 \%$ de la población del condado de Los Ángeles tiene orígenes hispanos (Oficina del Censo de EE.UU., 2010), por lo que la presencia y el uso del español en contacto con el inglés en esta zona es esperable. Además, la comunidad mexicana representa el $35.8 \%$ de la población hispana de la zona y la convierte en la más numerosa y representativa. Por último, y atendiendo a razones lingüísticas, los patrones entonativos del español mexicano presentan cualidades lo suficientemente características como para identificarlas con facilidad al compararlas con los patrones del inglés ${ }^{2}$. Los veinticuatro participantes fueron divididos en cuatro grupos homogéneos atendiendo a sus perfiles lingüísticos y sus observaciones personales:

1) Grupo control de inglés: ocho hablantes nativos de inglés (edad 26.7; DE 10.2) del condado de Los Ángeles. La lengua materna de estos participantes es el inglés y todos ellos han cursado español como su segunda lengua en el colegio.

2) Bilingües tempranos: ocho hablantes bilingües de inglés y español mexicano (edad 34.3; SD 12.2) que se mudaron de México a Los Ángeles antes de su adolescencia. Aunque la lengua materna de estos hablantes es el español, coinciden en que con el tiempo ha sido desplazada por usar el inglés con mayor regularidad. Este hecho es interesante ya que, a pesar de dicho desplazamiento y de que dos de ellos se consideraban 'pochos' (haciendo referencia a su español no estándar y menos fluido),

2 La sección 4 y el Apéndice muestran ejemplos y descripciones detalladas de estos patrones. 
estos hablantes consideran su herencia mexicana y el español como parte de su cultura e identidad.

3) Bilingües tardíos: cuatro hablantes bilingües de inglés y español mexicano (edad 39.5; DE 5.2) que se mudaron de México a Los Ángeles después de su adolescencia (edad de llegada 20; DE 2.1). La lengua materna de estos hablantes es el español, pero coinciden en que hoy en día usan el inglés de forma continua. Los participantes pertenecientes a este grupo también reconocen que el español es una parte sumamente importante en su identidad pero que el inglés tiene un valor instrumental añadido y que por ello intentan transmitir ambos a sus descendientes.

4) Grupo control de español: cuatro hablantes nativos de español (edad 26; DE 2.9) que han estado en Los Ángeles durante un breve periodo de tiempo (1.7 años, DE 0.5). El español es su lengua materna y aprendieron inglés como segunda lengua en el colegio en México.

Una vez presentados los cuatro grupos y sus características, mostraremos los tipos de frases producidas por todos ellos en la siguiente sección.

\section{METODOLOGÍA}

La recopilación de datos en la presente investigación sigue la metodología comúnmente empleada en otros estudios de entonación y prosodia (ver Prieto y Roseano 2010 para una selección de ejemplos en diferentes variedades del español). Dicha metodología, como es recalcado en Prieto y Frota (2015), está basada en el procedimiento "Discourse Completion Task" (Tarea de Compleción del Discurso) (Billmyer y Varghese 2000, Félix-Brasdefer 2010). En este tipo de tarea, el investigador presenta diferentes situaciones o contextos con el objeto de que los participantes respondan a los mismos con producciones naturales y espontáneas. La ventaja de utilizar este tipo de recopilación de datos es que el investigador puede controlar el tipo de producciones y su carga pragmática para así posibilitar hacer comparaciones más precisas entre sujetos y grupos. Los materiales y tipos de frases, aunque también están adecuados a los contextos en inglés, están basados en los expuestos en Prieto y Roseano (2010) para diferentes variedades de español. Los enunciados analizados en el presente estudio para ambos idiomas constaban de oraciones declarativas neutras sin foco (ej. Amanda compra 
bananas), oraciones imperativas tipo orden (ej. abre la ventana) y vocativos con la llamada de un nombre (ej. Marina) ${ }^{3}$.

El F0 (frecuencia fundamental) de todos los enunciados producidos por los veinticuatro participantes fue analizado con el programa de análisis acústico Praat (Boersma y Weenik 2016). Posteriormente, los movimientos tonales foco del estudio fueron etiquetados siguiendo el Modelo Métrico Autosegmental de notación prosódica, el sistema de transcripción ToBI (Tones and Breaks Indices) del español, así como estudios recientes en el campo (Beckman y Pierrehumbert 1986, Ladd 1996, Gussenhoven 2004, Beckman et al. 2005, Prieto y Roseano 2010, Hualde y Prieto 2015). Estos estudios asumen que las curvas melódicas están compuestas por tonos bajos ( $\mathrm{L}-$ low) y altos $(\mathrm{H}-$ high $)$ o sus combinaciones. Dichos tonos están asociados con sílabas acentuadas (marcadas con '*' - ejemplo L*) y fronteras prosódicas (marcadas con '\%” - ejemplo L\%). Siguiendo esta metodología podemos, con dos tonos ( $\mathrm{L}$ y H), describir y comparar los contornos melódicos, considerándolos una sucesión de elementos individuales (Ladd 2008).

A continuación, nos disponemos a dar más detalles de cada contexto pragmático. Dada la variedad de tonos y la necesidad de cubrirlos individualmente dependiendo de cada contexto, las siguientes subsecciones están divididas de la siguiente manera: 1) introducción con descripciones tonales encontradas en estudios previos, 2) etiquetaje tonal a emplear en el presente estudio, 3) resultados encontrados en cada grupo e idioma.

\section{CONTEXTOS PRAGMÁTICOS}

\subsection{ORACIONES DECLARATIVAS (ACENTOS TONALES PRENUCLEARES)}

Los acentos tonales prenucleares son aquellos que se encuentran antes de la última sílaba acentuada dentro de una frase. Atendiendo a descripciones previas del español, normalmente podemos encontrar tres tipos de acentos tonales (Sosa 1999, Face 2002, Beckman et al. 2002, Prieto y Face 2007,

\footnotetext{
3 El estudio también incluyó frases interrogativas absolutas y pronominales, pero dado que no se encontraron diferencias significativas entre grupos y debido a limitaciones de espacio, los resultados no son mostrados en este trabajo.
} 
Prieto y Roseano 2010, Hualde y Prieto 2015): 1) L+>H*: el F0 comienza a subir en la sílaba acentuada y el pico tonal se encuentra fuera de dicha sílaba (pico desplazado marcado con '>’). Este tipo de configuración es la más común en todas las variedades de español; 2) L+H*: el F0 comienza a subir en la sílaba acentuada y alcanza su máximo valor dentro de la misma sílaba. Este tipo de tonos son comunes en oraciones declarativas de foco estrecho; 3) $\mathrm{L}^{*}+\mathrm{H}$ : el F0 se mantiene bajo durante la sílaba acentuada y comienza a subir posteriormente.

En el caso del inglés, tradicionalmente se ha coincidido en que los tonos prenucleares en oraciones declarativas son altos $\left(\mathrm{H}^{*}\right)$, si bien la terminología $\mathrm{L}+\mathrm{H}^{*}$ también se ha empleado para movimientos de $\mathrm{F} 0$ similares (Pierrehumbert 1980, 2000, Pitrelli et al. 1994, Xu y Xu 2005, Ladd y Schepman 2003, Girand, 2006). Por su parte, Garding y Arvanity (2007) proponen que en el español que nos atañe (California del Sur) existe una distinción entre $\mathrm{H}^{*}, \mathrm{~L}+\mathrm{H}^{*}$ y $\mathrm{L}^{*}+\mathrm{H}$, donde la descripción de $\mathrm{L}^{*}+\mathrm{H}$ coincide con la etiqueta tonal $\mathrm{L}+>\mathrm{H}^{*}$ anteriormente propuesta para el español ${ }^{4}$. Con el objeto de usar un etiquetaje común y facilitar las comparaciones entre grupos e idiomas se consideraron los tonos recopilados en la Tabla $1^{5}$ :

\begin{tabular}{|c|c|c|}
\hline Tono & Representación & Descripción \\
\hline $\mathrm{L}+>\mathrm{H}^{*}$ & & $\begin{array}{l}\text { Comienzo de la subida del F0 en la sílaba } \\
\text { acentuada con un pico tonal fuera de dicha sílaba }\end{array}$ \\
\hline $\mathrm{L}^{*+\mathrm{H}}$ & & $\begin{array}{l}\text { Tono bajo en la sílaba acentuada seguido de una } \\
\text { subida en la sílaba posterior }\end{array}$ \\
\hline $\mathrm{H}^{*}$ & & F0 alto en la sílaba acentuada \\
\hline
\end{tabular}

Tabla 1: Tonos en acentos tonales prenucleares en frases declarativas

$4 \quad$ Ver Hualde y Prieto (2016) para una discusión más detallada sobre la necesidad de crear un alfabeto prosódico internacional para solucionar inconsistencias de etiquetaje como estas.

5 En los datos no se encontraron ejemplos de $\mathrm{L}+\mathrm{H}^{*}$ probablemente porque ninguna de las situaciones requería la producción de frases declarativas con foco estrecho. Ejemplos de otros tonos se recogen en el Apéndice. 
El número total de acentos tonales en posición prenuclear analizados fue de 145 (74 en español y 71 en inglés). La Figura 1 presenta los porcentajes de las producciones encontradas en los cuatro grupos y ambos idiomas. Las barras sólidas muestran los tonos esperados en ambos idiomas mientras que las barras con patrones cuadriculados corresponden a los tonos típicos del inventario del inglés.

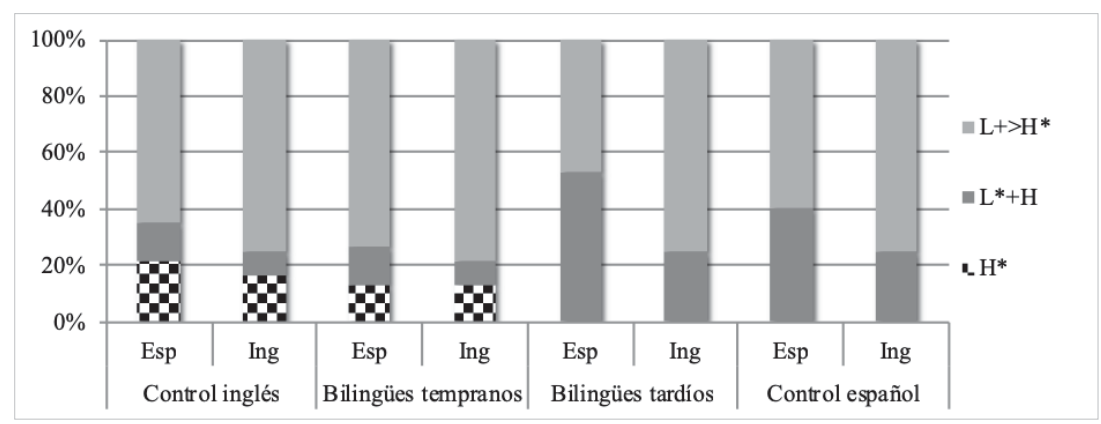

Figura 1: Tonos por grupo e idioma en acentos tonales pre-nucleares en producciones declarativas

Como se puede apreciar, todos los grupos produjeron tonos $\mathrm{L}+>\mathrm{H}^{*}$ y $\mathrm{L}^{*}+\mathrm{H}$; Sin embargo, el tono alto $\left(\mathrm{H}^{*}\right)$ típico del inglés solo se encontró en los dos primeros grupos sin hacer distinción entre idiomas (Control inglés: $21.4 \%$ en español, $16.7 \%$ en inglés y bilingües tempranos: $13.3 \%$ en español y $13 \%$ en inglés). Estos resultados nos hacen pensar que la temprana exposición al inglés por parte del grupo de bilingües tempranos ha permitido que estos hablantes hayan incorporado dicho tono a su inventario tonal al igual que el grupo control de inglés. En contraste, el grupo de bilingües tardíos y el grupo control español solo haría uso de tonos propios del español (y compartidos con el inglés).

\subsection{ORACIONES DECLARATIVAS \\ (ACENTOS TONALES NUCLEARES Y TONOS DE FRONTERA)}

Los acentos nucleares son aquellos situados al final de la frase y normalmente se caracterizan por ser percibidos como los más prominentes (Hualde 2005). En muchas variedades de español, incluyendo el español de México, es común encontrar acentos nucleares con una bajada continua o sin movimientos ascendentes claros ( $\mathrm{L}^{*} \mathrm{~L} \%$ ) o con una subida de F0 que comienza y termina dentro de la sílaba acentuada seguida de una 
bajada ( $\left.\mathrm{L}+\mathrm{H}^{*} \mathrm{~L} \%\right)$. Sin embargo, una de las características más notables en la entonación del español mexicano es el uso de subidas de F0 más pronunciadas $\left(\mathrm{L}+{ }_{i} \mathrm{H}^{*} \mathrm{~L} \%\right.$ - donde ' $i$ ' indica un pico más alto de lo normal) o incluso de tonos de frontera no descendentes $\left(\mathrm{L}+\mathrm{H}^{*} ! \mathrm{H} \%\right.$ - donde '! $\mathrm{H} \%$ ', indica que el tono final es medio) (Matluck 1951, Quilis 1993, Sosa 1999, Willis 2005, Martín Butragueño 2004, 2006, De la Mota et al. 2010, RoblesPuente 2011, Hualde y Prieto 2015).

En el caso del inglés, los tonemas (combinación de acento nuclear y tono de frontera) de las frases declarativas tradicionalmente se han etiquetado como H* (Pierrehumbert 1980, Beckman y Pierrehumbert 1986, Beckman y Hirschberg 1994). Sin embargo, las descripciones y representaciones de dichos movimientos tonales coinciden con las que en español se representan como $\mathrm{L}+\mathrm{H}^{*} \mathrm{~L} \%$. De hecho, al igual que en español, estos acentos nucleares han sido descritos como notablemente más bajos que los pre-nucleares hasta el punto de que en algunos casos pueden carecer de una subida o incluso presentar una bajada $\left(\mathrm{L}^{*} \mathrm{~L} \%\right)$. Nuevamente, con la intención de hacer uso de descripciones homogéneas y comparables, se emplearon las etiquetas recogidas en la Tabla 2 para ambos idiomas ${ }^{6}$ :

\begin{tabular}{|l|l|l|}
\hline Tonema & Representación & Descripción \\
\hline $\mathrm{L} * \mathrm{~L} \%$ & & $\begin{array}{l}\text { Bajada continua del F0 o falta de movimientos } \\
\text { seguida de un tono de frontera bajo }\end{array}$ \\
\hline $\begin{array}{l}\mathrm{L}+\mathrm{H}^{*} \\
\mathrm{~L} \%\end{array}$ & & $\begin{array}{l}\text { Subida del F0 en la sílaba acentuada alcanzando su } \\
\text { valor máximo dentro de dicha sílaba y seguida de } \\
\text { una bajada por un tono de frontera bajo (diferencia } \\
\text { de hasta 3 semitonos) }\end{array}$ \\
\hline $\begin{array}{l}\mathrm{L}+{ }_{i} \mathrm{H}^{*} \\
\mathrm{~L} \%\end{array}$ & $\begin{array}{l}\text { Subida del F0 en la sílaba acentuada alcanzando su } \\
\text { valor máximo dentro de dicha sílaba y seguida de } \\
\text { una bajada por un tono de frontera bajo (diferencia } \\
\text { de más de 3 semitonos) }\end{array}$ \\
\hline
\end{tabular}

6 Siguiendo a Butragueño (2006), la distinción entre $\mathrm{L}+\mathrm{H} * \mathrm{~L} \%$ y L $+{ }_{i} \mathrm{H}^{*} \mathrm{~L} \%$ viene dada por la diferencia en semitonos entre el valor más bajo y el más alto de la sílaba acentuada. 


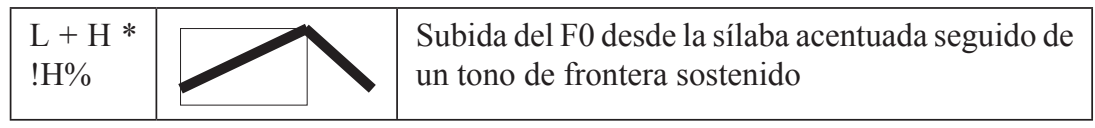

Tabla 2: Tonos en acentos tonales nucleares y tonos de frontera en frases declarativas

Se analizaron un total de 175 combinaciones de acentos nucleares y tonos de frontera (109 en español y 66 en inglés). La distribución de los tonos dependiendo del grupo y del idioma se pueden ver en la Figura 2. Las barras sólidas corresponden a tonos compartidos por ambos idiomas mientras que las barras con patrones a rayas corresponden a los tonos típicos del español mexicano.

Las configuraciones con una caída continua ( $\left.\mathrm{L}^{*} \mathrm{~L} \%\right)$ o con una subida leve seguida de un tono de frontera bajo $\left(\mathrm{L}+\mathrm{H}^{*} \mathrm{~L} \%\right)$ se produjeron en todos los grupos e idiomas, si bien la primera configuración fue la más encontrada (control inglés $95.5 \%$ en español y $96 \%$ en inglés; bilingües tempranos $72.5 \%$ en español y $85 \%$ en inglés; bilingües tardíos $91.4 \%$ en español y $58.4 \%$ en inglés; control español $62.5 \%$ en español y $50 \%$ en inglés). Las configuraciones más características del español mexicano $\left(\mathrm{L}+\mathrm{H}^{*} ! \mathrm{H} \%\right.$ y $\left.\mathrm{L}+{ }_{i} \mathrm{H} \mathrm{L} \%\right)$ no se observaron en el grupo control de inglés pero sí en los tres grupos cuya lengua materna era el español $(\mathrm{L}+\mathrm{H} *$ ! $\mathrm{H} \%$ : bilingües tempranos $12.5 \%$ en español y $10 \%$ en inglés; bilingües tardíos $16.6 \%$ en inglés; control español $25 \%$ en español y $25 \%$ en inglés. $\mathrm{L}+{ }_{i} \mathrm{H} \mathrm{L} \%$ : bilingües tempranos $2.5 \%$ en español y $10 \%$ en inglés; bilingües tardíos $8.6 \%$ en español y $25 \%$ en inglés; control español $12.5 \%$ en español y $16.6 \%$ en inglés).

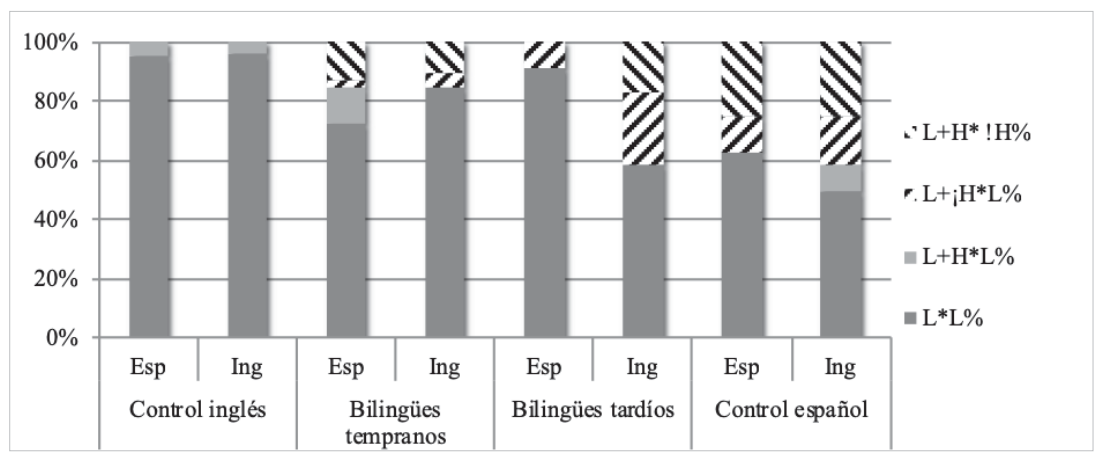

Figura 2: Tonemas por grupo e idioma en producciones declarativas 


\subsection{ORACIONES IMPERATIVAS}

El inglés y el español muestran una clara diferencia gramatical en las frases imperativas. Mientras que el inglés hace una clara distinción entre éstas y las frases declarativas, en español ambas pueden resultar idénticas. Una frase como 'abre la ventana' puede tener un valor declarativo en el que se comparte una información o puede ser una petición para que alguien haga algo. En inglés, por su parte, tendríamos un contraste entre ambos tipos de frases por el uso de sujetos explícitos o por las terminaciones verbales (I open the window vs. He/she opens the window vs. open the window). Lo interesante de esta ambigüedad en español es que en muchos casos no está resuelta por medio de la entonación. De hecho, múltiples estudios han equiparado los patrones tonales de las frases declarativas y las imperativas (Kvavik 1988, Willis 2002, Robles-Puente 2011), si bien es cierto que también se han encontrado diferencias entre las frases producidas en los dos contextos pragmáticos (de la Mota et al. 2010, Ortiz et al. 2010, RoblesPuente 2011). Estas diferencias incluyen valores de F0 más elevados en las frases imperativas así como acentos nucleares con picos notablemente más altos (denominados en muchos casos 'upstep' - $\mathrm{L}+{ }_{i} \mathrm{H}^{*} \mathrm{~L} \%$ ). En menor medida también se han encontrado imperativos con picos tempranos en acentos tonales en posición prenuclear $\left(\mathrm{L}+\mathrm{H}^{*}\right)$ así como tonos de frontera altos (H\%) (Willis 2002 y Robles-Puente 2011). En el caso del inglés no hay descripciones detalladas de producciones imperativas siguiendo el Modelo Métrico Autosegmental de notación prosódica. Sin embargo, hay reportes de imperativos que acaban tanto con tonos bajos como con tonos altos dependiendo del grado de amabilidad. Estos últimos normalmente son presentados como preguntas (Bolinger 1989, Culpeper et al. 2003, Gimson y Cruttenden 2001, Leech 2004).

Se analizaron un total de 84 tonemas imperativos (36 en español y 48 en inglés). Teniendo en cuenta la tendencia a compartir melodías entre las frases declarativas y las imperativas y basándonos en los resultados obtenidos, las etiquetas empleadas para la descripción de los tonemas imperativos serán los mismos que en la Tabla $2\left(\mathrm{~L}^{*} \mathrm{~L} \%, \mathrm{~L}+\mathrm{H}^{*} \mathrm{~L} \%, \mathrm{~L}+{ }_{i} \mathrm{H}^{*} \mathrm{~L} \%, \mathrm{~L}+\mathrm{H}^{*}\right.$ ! $\mathrm{H} \%$ ). Siendo conservadores, asumiremos que los patrones con una subida acentuada del F0 en posición nuclear $\left(\mathrm{L}+{ }_{i} \mathrm{H}^{*} \mathrm{~L} \%\right)$ pueden ser también propios de los imperativos del inglés como consecuencia de una fuerza ilocutiva extra. De esta forma, los tonos finales sostenidos $\left(\mathrm{L}+\mathrm{H}^{*} ! \mathrm{H} \%\right)$ serían los únicos exclusivos del español mexicano y estarían ejemplificados con barras con patrones a rayas en la Figura 3. Las barras sólidas muestran los tonos que son parte de los inventarios tonales tanto del inglés como del español. 
Observando la Figura 3 podemos notar que todos los tonemas son usados por los cuatro grupos excepto $\mathrm{L}+\mathrm{H}^{*}{ }_{i} \mathrm{H} \%$. Esta melodía se encuentra en las producciones en español de los grupos bilingües tempranos y tardíos, así como en las producciones en ambos idiomas del grupo control de español. Nuevamente los resultados indican que los patrones propios del español mexicano parecen estar presentes únicamente en los hablantes que tienen este idioma como lengua materna. Cabe destacar que, a diferencia de otros tonos exclusivos, ambos grupos bilingües presentan el tono típico del español mexicano únicamente en ese idioma, si bien es difícil concluir que es una elección consciente dado el bajo número de ejemplos.

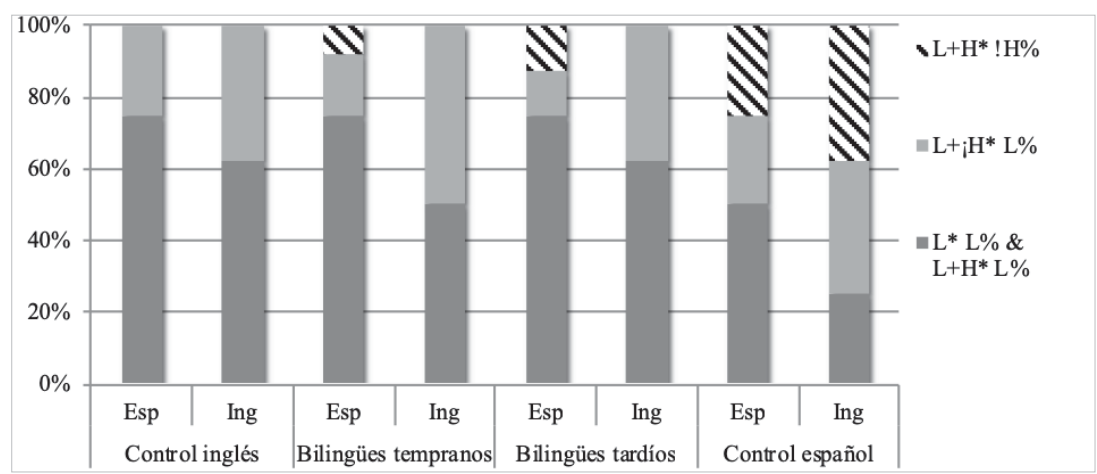

Figura 3: Tonemas por grupo e idioma en producciones imperativas

\subsection{VOCATIVOS}

Según Prieto y Roseano (2010), los vocativos pueden desempeñar varias funciones (llamar a alguien, mandar, etc.) y su configuración más común y neutral en español es L+H* $! \mathrm{H} \%$. En algunos casos y dependiendo del grado de insistencia pueden presentar también una configuración igual a la de las oraciones declarativas $\left(\mathrm{L}+\mathrm{H}^{*} \mathrm{~L} \%\right)$, como una pregunta $\left(\mathrm{L}+\mathrm{H}^{*} \mathrm{H} \%\right)$ o picos desplazados por un tono de frontera complejo (L+H* $\mathrm{HL} \%)$. En el caso del inglés, Liberman (1975), Ladd (1978, 1980), Pierrehumbert (1980) y Gussenhoven (2004) analizan el denominado canto vocativo (vocative chant) como una subida seguida de una bajada y un tono sostenido. Aunque su etiquetaje varía dependiendo de los autores, su descripción corresponde con la etiqueta $\mathrm{L}+\mathrm{H}^{*} ! \mathrm{H} \%$ anteriormente propuesta para el español. La Tabla 3 resume los tonemas posibles en las producciones vocativas y la Figura 4 recoge los resultados de las 84 producciones analizadas (36 en español y 48 en inglés). 


\begin{tabular}{|l|l|l|}
\hline Tonema & Representación & Descripción \\
\hline $\mathrm{L}+\mathrm{H}^{*} \mathrm{~L} \%$ & & $\begin{array}{l}\text { Subida del F0 en la sílaba acentuada alcanzando } \\
\text { su valor máximo dentro de dicha sílaba y seguida } \\
\text { de una bajada por un tono de frontera bajo }\end{array}$ \\
\hline $\begin{array}{l}\mathrm{L}+\mathrm{H} * \\
\mathrm{HL} \%\end{array}$ & & $\begin{array}{l}\text { Subida del F0 en la sílaba acentuada alcanzando } \\
\text { su valor máximo fuera de dicha sílaba y seguida } \\
\text { de una bajada por un tono de frontera bajo }\end{array}$ \\
\hline $\begin{array}{l}\mathrm{L}+\mathrm{H} * \\
\mathrm{H} \%\end{array}$ & & $\begin{array}{l}\text { Subida del F0 desde la sílaba acentuada seguido } \\
\text { de un tono de frontera sostenido }\end{array}$ \\
\hline $\begin{array}{l}\mathrm{L}+\mathrm{H} * \\
\mathrm{H} \%\end{array}$ & & Subida continua del F0 desde la sílaba acentuada \\
\hline
\end{tabular}

Tabla 3: Tonos en acentos tonales nucleares y tonos de frontera en frases vocativas

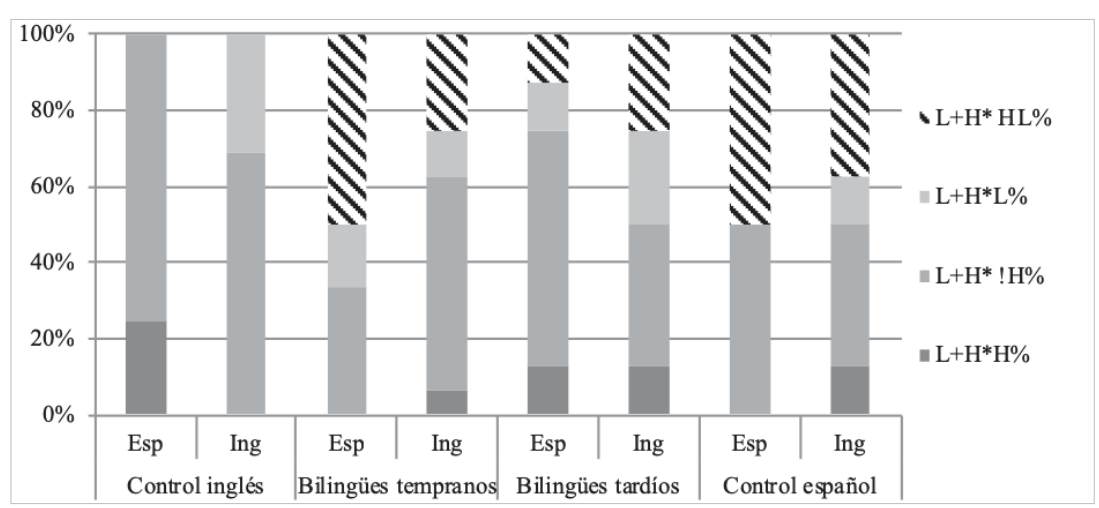

Figura 4: Tonemas por grupo e idioma en producciones vocativas

Basándonos en los porcentajes de la Figura 4 podemos concluir que el tono con el pico desplazado reportado para el español ( $\left.\mathrm{L}+\mathrm{H}^{*} \mathrm{HL} \%\right)$ es el único no encontrado en las producciones del grupo control de inglés. En dicho grupo el denominado 'canto vocativo' fue la melodía más común en las producciones de ambos idiomas. En el resto de los grupos la melodía $\mathrm{L}+\mathrm{H}^{*}$ HL\% propia del español tuvo una presencia notable (bilingües tempranos $50 \%$ en español y $25 \%$ en inglés; bilingües tardíos $12.5 \%$ en inglés y $25 \%$ en español; control español $50 \%$ en español y $37.55 \%$ en inglés). 


\section{RESULTADOS GENERALES}

En las secciones anteriores se han analizado 488 patrones entonativos producidos por los cuatro grupos (Control inglés, bilingües tempranos, bilingües tardíos y control español) en los dos idiomas (español e inglés) en tres contextos pragmáticos diferentes (oraciones declarativas neutras, imperativos tipo orden y vocativos). Atendiendo a esos resultados y haciendo una recopilación de los mismos, podemos configurar la Tabla 4, la Figura 5 y la Figura 6.

\begin{tabular}{|l|l|l|}
\hline Contexto & Tonos compartidos & Tonos exclusivos \\
\hline $\begin{array}{l}\text { Declarativa } \\
\text { (acento pre-nuclear) }\end{array}$ & $\begin{array}{l}\mathrm{L}+\mathrm{H} \\
\mathrm{L}+>\mathrm{H}^{*}\end{array}$ & $\mathrm{H}$ (ing.) \\
\hline $\begin{array}{l}\text { Declarativa } \\
\text { (tonema) }\end{array}$ & $\begin{array}{l}\mathrm{L} * \mathrm{~L} \% \\
\mathrm{~L}+\mathrm{H}^{*} \mathrm{~L} \%\end{array}$ & $\begin{array}{l}\mathrm{L}+\mathrm{H}^{*} \mathrm{~L} \% \text { (esp.) } \\
\mathrm{L}+\mathrm{H}^{*} ! \mathrm{H} \% \text { (esp.) }\end{array}$ \\
\hline $\begin{array}{l}\text { Imperativo } \\
\text { (tonema) }\end{array}$ & $\begin{array}{l}\mathrm{L} * \mathrm{~L} \% \\
\mathrm{~L}+\mathrm{H}^{*} \mathrm{~L} \% \\
\mathrm{~L}+\mathrm{H}^{*} \mathrm{~L} \%\end{array}$ & $\mathrm{~L}+\mathrm{H}^{*} ! \mathrm{H} \%$ (esp.) \\
\hline $\begin{array}{l}\text { Vocativo } \\
\text { (tonema) }\end{array}$ & $\begin{array}{l}\mathrm{L}+\mathrm{H}^{*} \mathrm{~L} \% \\
\mathrm{~L}+\mathrm{H}^{*} ! \mathrm{H} \%\end{array}$ & $\mathrm{~L}+\mathrm{H}^{*} \mathrm{HL} \%$ (esp.) \\
\hline
\end{tabular}

Tabla 4: Tonos por contexto pragmático

La Tabla 4 nos muestra la diferenciación entre los tonos que son exclusivos de uno de los dos idiomas o aquellos que son compartidos entre ambos mientras que la Figura 5 y la Figura 6 presentan la distribución de los mismos dependiendo del grupo. Ha de notarse que en ningún contexto las frases analizadas contenían al mismo tiempo tonos únicamente propios del inglés y del español, por ello los resultados son presentados y analizados individualmente. 


\section{Tonos del inglés}

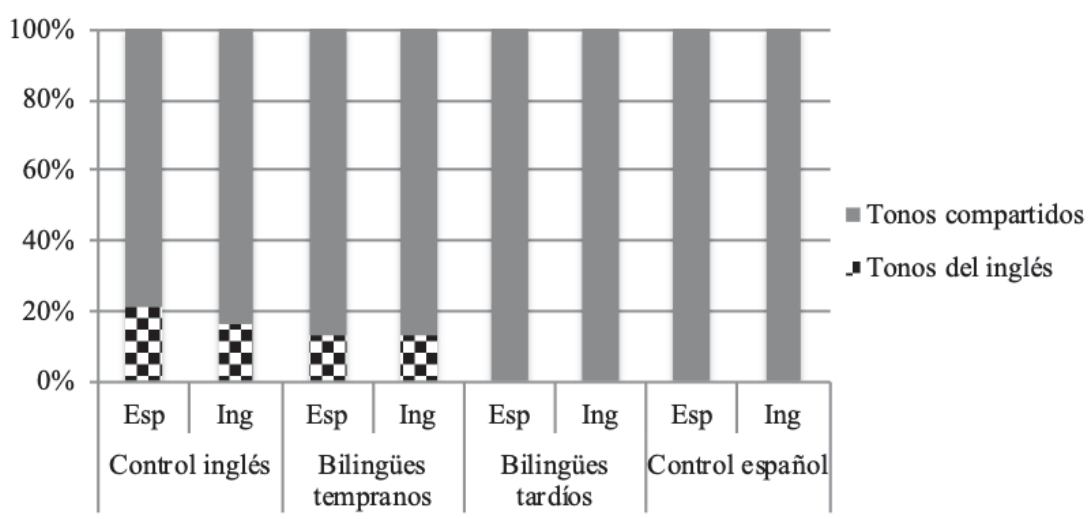

Figura 5: Porcentaje de tonos del inglés por grupo e idioma

La Figura 5 coincide con la Figura 1, ya que $\mathrm{H}^{*}$ es el único tono identificado como exclusivo del inglés. Dicho tono se encuentra en los acentos tonales prenucleares de las oraciones declarativas. Aunque el número de producciones con este tipo de melodía no ha sido muy elevado por la opción de usar tonos alternativos, se puede observar claramente que hay una diferencia entre los dos primeros grupos y los dos últimos. Una prueba de chi-cuadrado encontró diferencias entre grupos en el uso general de este tono $\mathrm{X}^{2}(3)=9.88, p=.020$ mientras que una serie de comparaciones por pares post-hoc demostró que había diferencias significativas entre los dos primeros grupos y los dos últimos. Ninguno de los grupos mostró diferencias significativas dependiendo del idioma. No es de extrañar que el grupo control de inglés haga uso de $\mathrm{H}^{*}$ en su idioma (16.7\%) ya que es parte de su inventario melódico. Además, su uso se extiende también al de su segunda lengua (español -21.4\%). Un resultado más interesante es que el grupo de bilingües tempranos, aun cuando su lengua materna es el español, parecen haber introducido $\mathrm{H}^{*}$ no solo en sus producciones del inglés $(13 \%)$ sino también en las del español (13.3\%). Por su parte, el grupo de bilingües tardíos y el grupo control de español parecen no haber incorporado $\mathrm{H}^{*}$ a sus producciones en ninguna de las dos lenguas y optan por usar otras melodías. 


\section{Tonos del español}

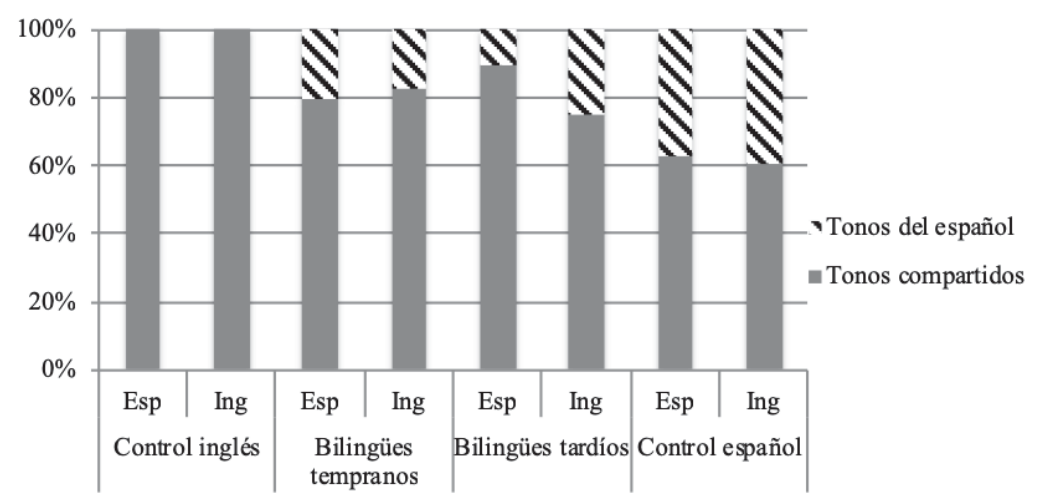

Figura 6: Porcentaje de tonos del español por grupo e idioma

La Figura 6 captura el uso de los tonos que han sido identificados como propios del español pero no del inglés $\left(\mathrm{L}+{ }_{i} \mathrm{H}^{*} \mathrm{~L} \%\right.$ en declarativas, $\mathrm{L}+\mathrm{H}^{*}$ $! \mathrm{H} \%$ en declarativas e imperativos y $\mathrm{L}+\mathrm{H}^{*} \mathrm{HL} \%$ en vocativos). Hay un claro contraste entre el grupo de control de inglés ( $0 \%$ de tonos del español) y los tres grupos cuya lengua materna es el español (bilingües tempranos $20.3 \%$ en español, $17.3 \%$ en inglés; bilingües tardíos $10.3 \%$ en español y $25 \%$ en inglés; grupo control de español $37.5 \%$ en español y $39.3 \%$ en inglés). A su vez, se puede notar un mayor uso de estos tonos por parte del grupo que menos tiempo ha pasado en contacto con el inglés (grupo control de español). Estas observaciones fueron corroboradas por una prueba de chi-cuadrado que encontró diferencias entre grupos en el uso general de los tonos del español $\mathrm{X}^{2}(3)=40.35, p<.000$. Las comparaciones por pares posteriores mostraron diferencias significativas (todas $p<.01$ ) entre el grupo control de inglés y el resto de grupos, así como entre los dos grupos de bilingües y el grupo control de español. Al igual que con los tonos del inglés, ningún grupo mostró diferencias significativas en el uso de los tonos del español, dependiendo del idioma, por lo que podemos concluir que hay una falta de acomodación en este aspecto. 


\section{CONCLUSIONES}

El principal objetivo de este trabajo es aportar información sobre cómo los inventarios tonales de bilingües de español e inglés pueden verse afectados por la existencia de dos gramáticas en situaciones de contacto de lenguas. Sin embargo, los resultados obtenidos nos llevan a observaciones adicionales más generales, que son también relevantes en el campo de la prosodia y la entonación.

En primer lugar, el análisis acústico del F0 de las producciones recogidas ha demostrado que, a pesar de los diferentes etiquetajes fonológicos usados por diferentes autores en ambas lenguas en el pasado, el español y el inglés comparten un número notable de melodías. Esta observación refuerza la propuesta reciente de Hualde y Prieto (2016) de construir un alfabeto prosódico internacional que permita una mayor consistencia a la hora de hacer comparaciones entre idiomas. A pesar de las similitudes entre muchos movimientos tonales, las comparaciones entre los cuatro grupos de estudio nos permiten ver también la falta de homogeneidad entre hablantes.

Los grupos control de español e inglés proporcionan una base que nos permite ver que los tonos exclusivos de cada idioma $\left(\mathrm{H}^{*}\right.$ para el inglés y $\mathrm{L}+{ }_{j} \mathrm{H}^{*} \mathrm{~L} \%, \mathrm{~L}+\mathrm{H}^{*} ! \mathrm{H} \%$ y $\mathrm{L}+\mathrm{H}^{*} \mathrm{HL} \%$ para el español dependiendo del contexto pragmático) son usados únicamente por los hablantes nativos de dicho idioma. A su vez, esos hablantes producen los tonos en ambos idiomas, poniendo de manifiesto un proceso de transferencia al nivel de la prosodia. Puede argumentarse que los grupos control deberían haber sido formados por hablantes monolingües puros, sin embargo, el analizar las dos lenguas simultáneamente nos aporta datos relevantes sobre los inventarios tonales de estos sujetos. Además, los resultados muestran que una potencial influencia de la segunda lengua en los grupos control ha debido ser mínima ya que siguen mostrando los patrones esperados en monolingües. Por ejemplo, el grupo control de español, a pesar de estar formado por sujetos que han pasado un breve periodo de tiempo en EE.UU., sigue mostrando entonaciones más propias del español mexicano. Esperaríamos que un grupo de monolingües sin ningún tipo de contacto con el inglés mostrara diferencias todavía más claras.

Los usos de $\mathrm{H}^{*}$ en acentos tonales prenucleares en frases declarativas por parte del grupo de bilingües tempranos, pero no en los otros dos grupos cuya lengua materna es el español, indican que una temprana exposición a un segundo idioma (en este caso el inglés) parece permitir una incorporación de los tonos del mismo. Además, dichos tonos se observan también en sus producciones del español, por lo que podemos argumentar que la 
segunda lengua ha podido tener una influencia en la primera. Este punto es especialmente relevante, ya que, aunque los sujetos pertenecientes a este grupo consideran el español como una parte importante de su identidad, esto no parece evitar la modificación del inventario tonal español con tonos del inglés. La aparición de melodías propias del español mexicano tanto en español como en el inglés del grupo bilingüe temprano, el grupo bilingüe tardío y el grupo control de español muestran el ejemplo contrario y cómo las propiedades entonativas de la lengua materna tienden a transferirse a la segunda lengua. Todas estas observaciones confirman que el dominio prosódico de la frase entonativa puede verse afectado cuando existen dos gramáticas alternativas (Selkirk 1980, 1984, Nespor y Vogel 1986, Hayes 1989, Schiering et al. 2007, Prieto 2006).

En el futuro sería óptimo llevar a cabo estudios paralelos con más sujetos en otras comunidades y variedades en contacto con el inglés para complementar los presentes resultados. Dada la situación especial de la comunidad mexicana en Los Ángeles, tanto por su número e historia como por su proximidad con México, nos podemos preguntar si encontraríamos resultados similares en comunidades más pequeñas o alejadas del país de origen. De la misma forma, es bien sabido que otras variedades de español muestran características entonativas diferentes a la mexicana. El estudio de dichas variedades con grupos similares a los del presente estudio nos permitiría comprender mejor cómo y por qué las características entonativas pueden verse afectadas en mayor o menor medida en situaciones de contacto.

\section{REFERENCIAS BIBLIOGRÁFICAS}

Alvord, Scott. 2006. Spanish intonation in contact: the case of Miami Cuban bilinguals. Tesis doctoral, University of Minnesota.

Beckman, Mary. E. y Julia Hirschberg. 1994. The ToBI Annotation Conventions [en línea]. Disponible en https://www.ling.ohio-state.edu/ tobi/ame_tobi/annotation_conventions. html [Consulta 05/02/2011].

Beckman, Mary E. y Janet Pierrehumbert. 1986. Intonational Structure in Japanese and English. Phonology Yearbook 3: 255-309.

Beckman, Mary. E., Manuel Díaz-Campos, Julia McGory y Terence Morgan. 2002. Intonation Across Spanish in the Tones and Break Indices Framework. Probus 14: 9-36.

Beckman, Mary E., Julia Hirschberg y Stefanie Shattuck Hufnagel. 2005. The original ToBI system and the evolution of the ToBI framework. En Sun-Ah Jun (ed.). Prosodic typology: The phonology of intonation and phrasing, pp. 9-54. New York: Oxford University Press.

Billmyer, Kristine y MANKa VARghese. 2000. Investigating instrument based pragmatic variability: Effects of enhancing discourse completion tests. Applied Linguistics 21 (4): 517-552. 
Boersma, Paul y David Weenink. 2016. Praat: doing phonetics by computer [en línea]. Disponible en http://www.fon.hum.uva.nl/praat/ [Consulta 06/01/2016].

Bolinger, Dwight. 1989. Intonation and Its Uses: Melody in Grammar and Discourse. Stanford: Stanford University Press.

CARTer, Phillip M. 2005. Quantifying Rhythmic Differences between Spanish, English, and Hispanic English. En Randall Gess y Edward J. Rubin (eds.). Theoretical and Experimental Approaches to Romance Linguistics: Selected Papers from the 34th Linguistic Symposium on Romance Languages (Current Issues in Linguistic Theory 272), pp. 63-75. Amsterdam, Philadelphia: John Benjamins.

Carter, Phillip M. y Tonya Wolford. 2016. Cross-generational prosodic convergence in South Texas Spanish. Spanish in Context 13: 29-52.

Colantoni, Laura y Jorge Gurlekian. 2004. Convergence and intonation: historical evidence from Buenos Aires Spanish. Bilingualism: Language and Cognition 7: 107-119.

Culpeper, Jonathan, Derek Bousfield y Anne Wichmann. 2003. Impoliteness revisited: With special reference to dynamic and prosodic aspects. Journal of Pragmatics 35: 1545-1579.

de-la-Mota, Carme, Pedro Martín Butragueño y Pilar Prieto. 2010. Mexican Spanish Intonation. En Pilar Prieto y Paolo Roseano (eds.). Transcription of Intonation of the Spanish Language, pp. 319-350. München: Lincom Europa.

Elordieta, Gorka. 2003. The Spanish intonation of speakers of a Basque pitch-accent dialect. Catalan Journal of Linguistics 2: 67-95.

Face, Timothy L. 2002. Intonational Marking of Contrastive Focus in Madrid Spanish. Munich: Lincom Europa.

FéliX-Brasdefer, J. Cesar. 2010. Data collection methods in speech act performance: DCTs, role plays, and verbal report. En Alicia Martínez-Flor y Esther Usó-Juan (eds.). Speech act performance: Theoretical, empirical and methodological issues, pp. 41-56. Amsterdam: John Benjamins.

Fought, Carmen. 2003. Chicano English in Context. Basingstoke, United Kingdom: Palgrave Macmillan.

Garding, Gina y Amalia Arvaniti. 2007. Dialectal Variation in the Rising Accents of American English. Papers in Laboratory Phonology IX: Change in Phonology 9: 547-576.

Gimson Alfred. C. y Alan Cruttenden. 2001. Gimson's Pronunciation of English. London: Edward Arnold.

Girand, Cynthia. 2006. Prenuclear Accentual Structure in Conversational English. Tesis doctoral, University of Colorado.

Gussenhoven, Carlos. 2004. The phonology of tone and intonation. Cambridge: Cambridge University Press.

HAYes, BRUCE. 1989. The prosodic hierarchy in meter. En Paul Kiparsky y Gilbert Youmans (eds.). Rhythm and Meter, Phonetics and Phonology 1: 201-260, New York: Academic Press.

Hualde, José Ignacio. 2005. The Sounds of Spanish. Cambridge, UK/NY: Cambridge University Press.

Hualde, Jose Ignacio y Pilar Prieto. 2015. Intonational variation in Spanish: European and American varieties. En Sonia Frota y Pilar Prieto (eds.). Intonational Variation in Romance, pp. 350-391. Oxford University Press.

2016. Towards an Internation Prosodic Alphabet (IPrA). Laboratory Phonology: Journal of the Association for Laboratory Phonology 7 (1): 1-25.

Kireva, Elena. 2013. Prosodic transfer in a contact variety: The case of Olivenza Spanish. Poster presentado en la conferencia Phonetics and Phonology in Iberia (PaPI 2013). Universidad de Lisboa, Lisboa, Portugal. 
Kvavik, Karen. 1988. Is there a Spanish imperative intonation? En Robert M. Hammond, y Melvyn C. Resnick (eds.). Studies in Caribbean Spanish Dialectology, pp. 35-49. Washington, D.C.: Georgetown University Press.

LADD, D. RoBert. 1978. Stylized intonation. Language 54: 517-540. 1980. The Structure of Intonational Meaning. Indiana University Press, Bloomington y Londres. 1996, 2008. Intonational Phonology. Cambridge: Cambridge University Press.

LAdD, D. Robert y Astrid SchePMAn. 2003. Sagging transitions between high pitch accents in English: experimental evidence. Journal of Phonetics 31: 81-112.

Leech, Geoffrey. N. 2004. Meaning and the English Verb. London: Pearson Education.

LiBERMAn, MARK. 1975. The Intonational System of English. Tesis doctoral no publicada, MIT. LIPSKI, JoHn M. 2008. Varieties of Spanish in the United States. Georgetown University Press.

Martín Butragueño, Pedro. 2004. Configuraciones circunflejas en la entonación del español mexicano. Revista de Filología Española 84 (2): 347-373.

2006. Proyección sintáctico-discursiva de la entonación circunfleja mexicana. En Concepción Company (ed.). El español en América. Diatopía, diacronía e historiografía. Homenaje a José G. Moreno de Alba en su 65 aniversario, pp. 35-63. UNAM, México.

MatLuCK, Joseph H. 1951. La pronunciación en el español del Valle de México. Tesis doctoral, UNAM.

Miglio, Viola, Stefan Gries y Michael Harris. 2013. New/given information in Mexican \& Chicano Spanish intonation. Poster presentado en la reunión 88 de la Linguistic Society of America, Minneapolis, Minnesota.

Nespor, Marina e Irene Vogel. 1986. Prosodic Phonology. Dordrecht: Foris.

O'Rourke, Erin. 2005. Intonation and language contact: A case study of two varieties of Peruvian Spanish. Tesis doctoral, University of Illinois at Urbana-Champaign.

Ortiz, Héctor, Marcela Fuentes y Llü̈sa Astruc. 2010. Chilean Spanish Intonation. En Pilar Prieto y Paolo Roseano (eds.). Transcription of Intonation of the Spanish Language, pp. 255-283. München: Lincom Europa.

Pierrehumbert, Janet. 1980. The phonology and phonetics of English intonation. Tesis doctoral, Indiana University. Bloomington: Indiana University Press.

2000. Tonal elements and their alignment. En Bruce Merle (ed.). Prosody: Theory and Experiment, pp. 11-36. Dordrecht/Boston/London: Kluwer.

Pitrelli, John, Mary. E Beckman y Julia Hirschberg. 1994. Evaluation of prosodic transcription labeling reliability in the ToBI framework. ICSLP94 1: 123-126.

Prieto, Pilar. 2006. Phonological Phrasing in Spanish. En Fernando Martínez-Gil y Sonia Colina (eds). Optimality-theoretic Advances in Spanish Phonology, pp. 39-60. Amsterdam/ Philadelphia: John Benjamins.

Prieto, Pilar y Sonia Frota (eds.). 2015. Intonational Variation in Romance. Oxford University Press.

Prieto, Pilar y Paolo Roseano. 2010. Transcription of Intonation of the Spanish Language. Lincom Europa: München.

Prieto, Pilar y Timothy Face. 2007. Rising accents in Castilian Spanish: a revision of SpToBI. En Gorka Elordieta y Marina Vigário (eds.). Journal of Portuguese Linguistics 6, 1 (special issue on Prosody of Iberian Languages), pp. 117-146.

Quilis, Antonio. 1993. Tratado de fonología y fonética españolas. Madrid: Gredos.

Robles-Puente, Sergio. 2011. Looking for the Spanish Imperative Intonation: Combination of Global and Pitch-Accent Level Strategies. En Scott M. Alvord (ed.). Selected Proceedings of the 5th Conference on Laboratory Approaches to Romance Phonology, pp. 153-164. Somerville, MA: Cascadilla Proceedings Project. 
2012. Two Languages, two intonations? Statements and Yes/No Questions in Spanish and Basque. En Rebeka Campos-Astorkiza Y Jon A. Franco, Jon (eds.). International Journal of Basque Linguistics and Philology (ASJU), 46 (1): 252-262.

Schiering, René, Kristine Hildebrandt y Balthasar Bickel. 2007. Crosslinguistic challenges for the prosodic hierarchy [en línea]. Disponible en http://www.autotyp.uzh.ch/download/ SchieringHildebrandtBickel2007.pdf [Consulta 04/04/2016].

SelKirK, Elisabeth O. 1980. The role of prosodic categories in English word stress. Linguistic Inquiry 11: 563-605.

1984. Phonology and Syntax: The Relation between Sound and Structure. Cambridge: MIT Press.

Shousterman, Cara. 2014. Speaking English in Spanish Harlem: The Role of Rhythm. University of Pennsylvania Working Papers in Linguistics 20 (2): 157-168.

Silva-Corvalán, Carmen. 1994. Language contact and change: Spanish in Los Angeles. (Oxford studies in language contact). Oxford: Clarendon.

Sosa, JuAn Manuel. 1999. La entonación del español: su estructura fónica, variabilidad y dialectología. Madrid: Cátedra.

Thomas, Erik y Holly A. Ericson. 2007. Intonational distinctiveness of Mexican American English. University of Pennsylvania Working Papers in Linguistics 13 (2): 193-205.

Thomason, SARAh G. y Terrence Kaufman. 1988. Language contact, creolization, and genetic linguistics. Berkeley: University of California Press.

WILLIS, ERIK W. 2002. Is there a Spanish imperative intonation revisited: Local considerations. Linguistics 40: 347-74.

2005. Tonal Levels in Puebla Mexico Spanish Declaratives and Absolute Interrogatives. En Randall Gess y Ed Rubin (eds.). Theoretical and Experimental Approaches to Romance Linguistics, pp. 351-363. Amsterdam: John Benjamins.

Xu, Yi y Ching Xu. 2005. Phonetic realization of focus in English declarative intonation. Journal of Phonetics 33: 159-197. 


\section{APÉNDICE}

Ejemplos de configuraciones tonales (las partes sombreadas indican

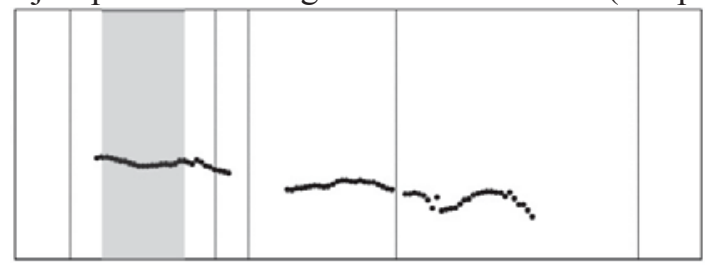

\begin{tabular}{|l|l|l|l|l|l|}
\hline & Amanda & is & buying & bananas & \\
\hline
\end{tabular}

las sílabas acentuadas relevantes):

$\mathrm{H}^{*}$ en posición prenuclear (declarativa)
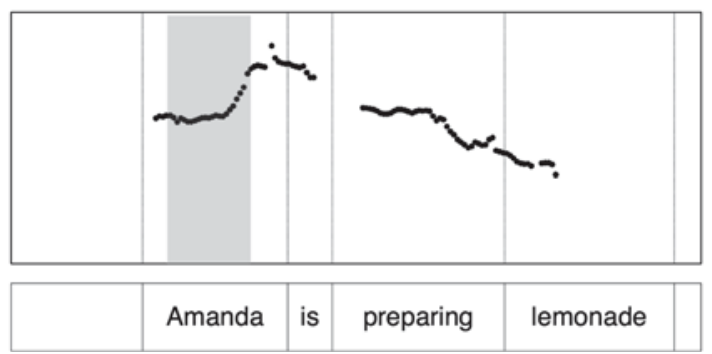

L*+H en posición prenuclear (declarativa)

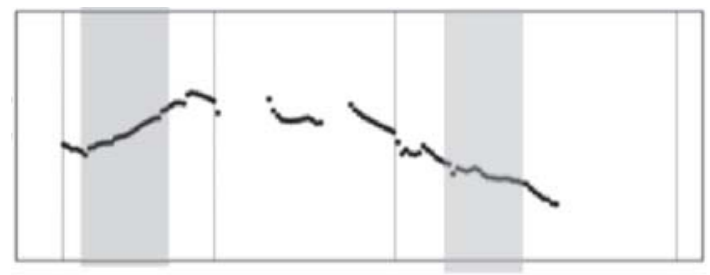

\begin{tabular}{|l|l|l|l|}
\hline & Amanda & compra & bananas \\
\hline
\end{tabular}

$\mathrm{L}+>\mathrm{H}^{*}$ en posición pre-nuclear y tonema L* L\% (declarativa)

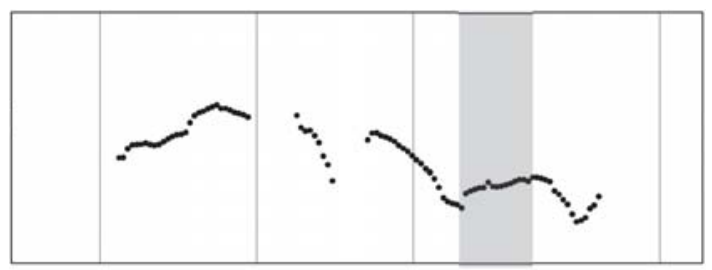

\begin{tabular}{|l|l|l|l|l|}
\hline & Amanda & compra & bananas & \\
\hline
\end{tabular}

Tonema L+H* L\% (declarativa) 


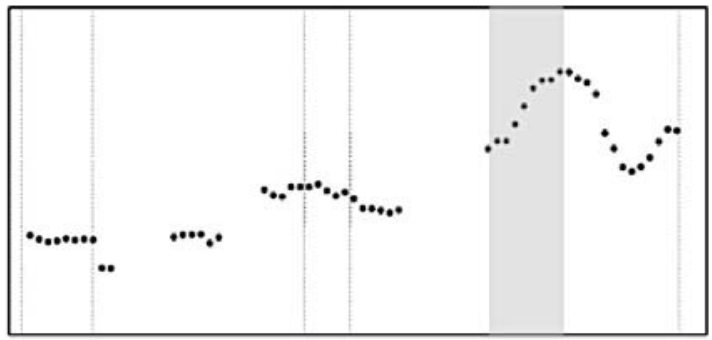

Tonema $\mathrm{L}+\mathrm{H}^{*} ! \mathrm{H} \%$

(declarativa)
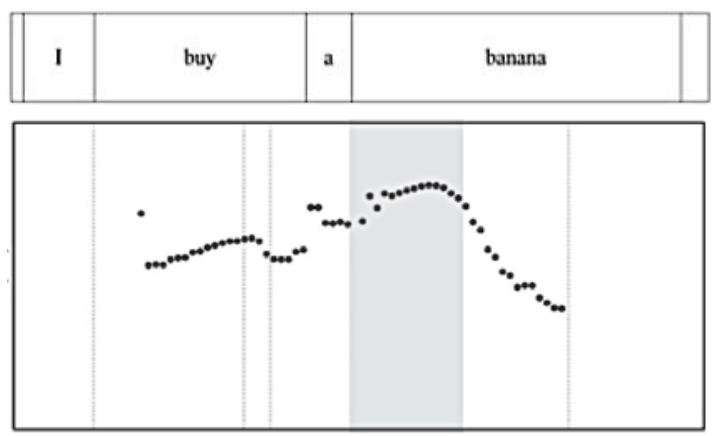

Tonema $\mathrm{L}+{ }_{\mathrm{i} H ~ \mathrm{~L} \%}$

(imperativo)

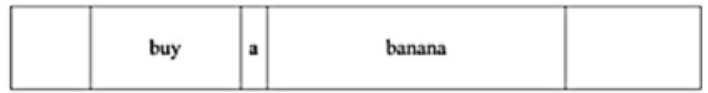

Tonema $\mathrm{L}+\mathrm{H}^{*}$

! $\mathrm{H} \%$ (vocativo)

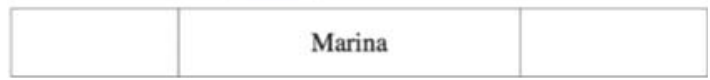

Tonema $\mathrm{L}+\mathrm{H}^{*} \mathrm{H} \%$ (vocativo)

\section{Marina}



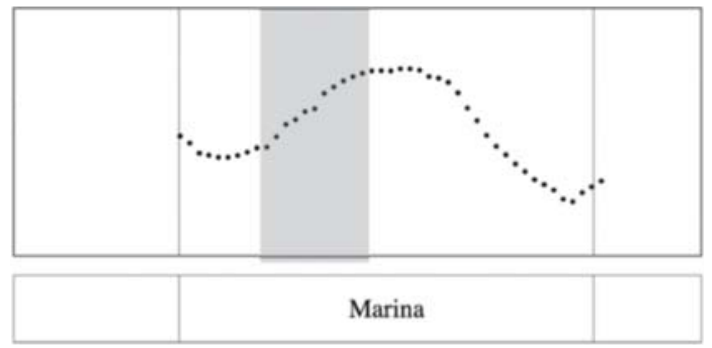

Tonema L $+\mathrm{H}^{*}$

HL\% (vocativo) 